\title{
Use of electronic health records to evaluate treatment pathways - a Common Data Model approach
}

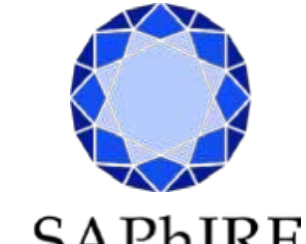

$\underline{\text { Tan Hui Xing', Cynthia Sung }}{ }^{1,2}$, Chahed Haroun ${ }^{1}$, Teo Chun Hwee Desmond', Ang Pei San', Tan Su Yin Doreen ${ }^{3}$, Dorajoo Sreemanee Raaj'

1 Vigilance \& Compliance Branch, Health Products Regulation Group, Health Sciences Authority, Singapore

2 Health Services and Systems Research, Duke-NUS Medical School, Singapore ${ }^{3}$ Department of Pharmacy, Khoo Teck Puat Hospital, Singapore

\section{INTRODUCTION}

Electronic medical records offer a potentially rich resource to uncover drug utilization patterns, and evaluate adherence to recommended treatment guidelines. This study demonstrates the use of a common data model (CDM) to harmonise electronic medical records (EMR) to investigate the pharmacological treatment patterns of patients newly diagnosed with diabetes, hypertension or depression.

\section{OBJECTIVES}

To transform data from an acute hospital in Singapore to the common data model (CDM) developed by the Observational Medical Outcomes Partnership (OMOP)

- To apply analytical tools on the data to uncover the pharmacological treatment patterns of patients newly diagnosed with diabetes mellitus, hypertension or depression.

\section{METHODOLOGY}

\section{Conversion of source data files to the OMOP CDM:}

- The data of $\sim 250,000$ patients who had visited a tertiary care hospital in Singapore from January 2013 to December 2016 were mapped onto the CDM schema, through a series of Extract, Transform, Load processes written in SQL. The data contained 1.1 million rows of diagnoses, 5.2 million rows of ordered medications and 15.5 million lab records.

Federated query on the transformed data:

- This query returned the sequences of drug exposure in patients newly diagnosed with diabetes, depression or hypertension. Treatment pathway comparisons were made using sunburst diagrams.

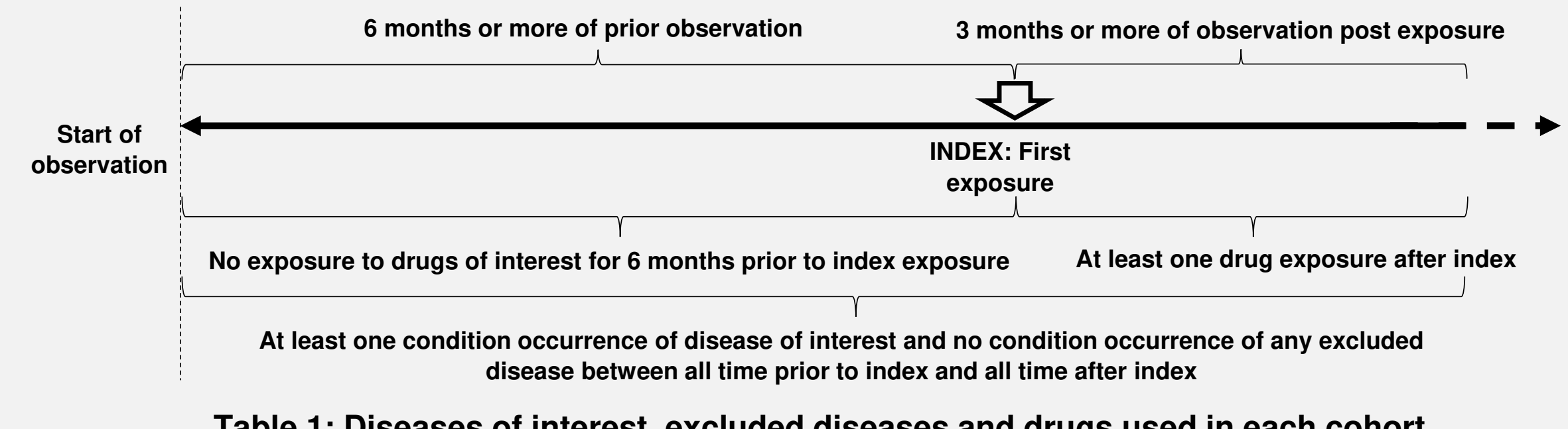

Table 1: Diseases of interest, excluded diseases and drugs used in each cohort

\begin{tabular}{|l|l|l|l|}
\hline Cohort & Disease of interest & Excluded disease & Drug classes included \\
\hline Diabetes & Diabetes mellitus & Findings related to pregnancy & $\begin{array}{l}\text { Insulins, biguanides, sulfonylureas, DPP4 inhibitors*, } \\
\text { alpha-glucosidase inhibitors, SGLT2 inhibitors^ }\end{array}$ \\
\hline Hypertension & Hypertensive disorder & Findings related to pregnancy & $\begin{array}{l}\text { Antihypertensives, diuretics, peripheral vasodilators, beta } \\
\text { blockers, calcium channel blockers, agents acting on the } \\
\text { renin-angiotensin-aldosterone system }\end{array}$ \\
\hline Depression & Depressive disorder & $\begin{array}{l}\text { Findings related to pregnancy, } \\
\text { bipolar I disorder, schizophrenia }\end{array}$ & Antidepressants \\
\hline
\end{tabular}

\section{RESULTS \& DISCUSSION}

Three cohorts of patients with depression $(n=251)$, hypertension $(n=3,175)$ and diabetes mellitus $(n=1,006)$ with their index prescriptions between 2015 and 2016 were identified.

\section{Diabetes Mellitus:}

Metformin was most often prescribed as the first medication (53.5\%). Sulfonylureas were the most common second line agent used in diabetes. Interestingly, among the agents used, glipizide was the most common (used in $43.3 \%$ of all patients), followed by tolbutamide $(7.1 \%)$ whereas arguably safer newer generation alternatives such as gliclazide and glimepiride were used less frequently, at 5.5 and $0.6 \%$, respectively. Sunburst charts of the treatment pathways also revealed the relative frequencies at which subsequent agents were used as second, third or fourth line therapies.

Hypertension and Depression: There was a more even distribution of medication use, with mirtazapine $(37.1 \%)$ and fluvoxamine $(19.1 \%)$ being the more commonly used first line medications for depression, and amlodipine $(22.1 \%)$ being the more commonly used first line medication for hypertension.

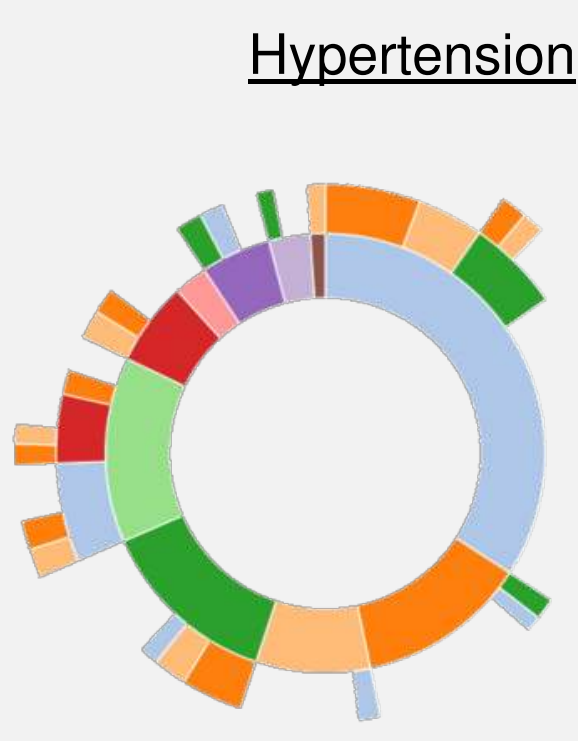

\section{CONCLUSION}

- There is considerable heterogeneity in treatment patterns for hypertension and depression, whereas for diabetes, metformin is the most common first line agent (used in $53.5 \%$ of patients).

- We showed that the same query previously used in a similar study ${ }^{1}$ on external data sources could be applied consistently to our dataset, demonstrating the feasibility of use of the CDM.

- These models provide drug regulators valuable insights on real world drug utilization patterns and adherence to recommended treatment guidelines.

\section{ACKNOWLEDGEMENTS}

This work was supported by a Strategic Positioning Fund grant from the Biomedical Research Council of the Agency for Science, Technology and Research of Singapore (SPF2014/001).

Reference 1. Hripcsak, G., et al. Observational Health Data Sciences and Informatics (OHDSI): Characterising treatment pathways at scale using the OHDSI network. Proc Natl Acad Sci U.S.A, 2016. 113(27):7329-36. 